\title{
Exploitation of the neural-hematopoietic stem cell niche axis to treat myeloproliferative neoplasms
}

\author{
Naoimh Herlihy, Claire N Harrison and Donal P McLornan \\ Department of Haematology, Guy's and St. Thomas' NHS Foundation Trust, London, UK \\ E-mail: CLAIRE HARRISON - claire.harrison@gstt.nhs.uk
}

doi:10.3324/haematol.2018.211896

M yeloproliferative neoplasms $(\mathrm{MPN})$ originate from a population of hematopoietic stem cells (HSCs) within the bone marrow (BM) that undergo clonal expansion as a result of factors both intrinsic and extrinsic to the cell. They are characterized by progressive marrow fibrosis, heterogeneous symptomatology, extramedullary hematopoiesis, splenomegaly, a propensity to both hemorrhage and thrombosis, and an inherent risk of transformation to acute myeloid leukemia (AML). Recently, there has been an increasing focus on the role of the BM HSC niche in MPN development and disease maintenance. ${ }^{1}$ This niche encompasses complex cellular and signaling networks with multiple interactions and 'cross-talk' between HSC, mesenchymal cells (MSC), perivascular cells identified as chemokine ( $\mathrm{C}-\mathrm{X}-\mathrm{C}$ motif) ligand 12 (CXCL12)-abundant reticular (CAR) cells, osteolineage-derived cells and sinusoidal endothelial cells, amongst others ${ }^{1-3}$ (Figure 1). Importantly, the acquisition of gain-of-function mutations such as JAK2-V617F by MPN HSCs or other cells can result in an alteration of the niche to favor clonal expansion at the expense of background normal HSCs. ${ }^{4}$ For example, Zhan et al. recently suggested that JAK2-V617F mutant endothelial cells in the vascular niche promote clonal expansion of JAK2-V617F HSCs at the expense of wild-type progenitors. ${ }^{5}$ In chronic myeloid leukemia (CML), one study found BCR/ABL transgenic mice create a self-reinforcing leukemic niche that impairs normal hematopoiesis, favors leukemic stem cell function and contributes to development of BM fibrosis by stimulating MSCs to overproduce osteoclastic stem cells. ${ }^{6}$ Conversely, there is also mounting evidence that targeted disruption of the HSC niche may result in development of MPN, for example defective Notch activation ${ }^{7}$ and ablation of the retinoic acid receptor gamma $a^{8}$ and retinoblastoma genes ${ }^{9}$ have been evaluated in murine models, suggesting that genetic mutations in the niche itself can also drive the malignant process. Little was known about the role of the MSC population in MPN-pathogenesis and maintenance and data were often conflicting. Avanzini et al. described how MPN-BM-derived MSC exhibited decreased proliferative and osteogenic capacity whereas Martinaud et al. suggested an enhanced and persistent increase in osteogenic abilities coupled with an altered secretome and transcriptome in primary myelofibrosis (PMF)-derived BM-MSC. ${ }^{10,11}$ More recently, Ramos et al. demonstrated that BM-MSC derived from JAK2-mutated MPN patients favored maintenance of clonal hematopoietic cells. ${ }^{12}$ Targeting of this population, in particular increasing the 'beneficial' MSC subgroup, is hence of potential interest.

Nearly a decade ago, Méndez-Ferrer et al. originally described how Nestin-positive $\left(^{+}\right)$MSC are essential components of the HSC niche, containing all of the BM colony-forming-unit fibroblastic activity and having the ability to function as so-called 'niche-forming' cells. ${ }^{13,14}$ Nestin is a type VI intermediate filament protein and functions as a major component of the cytoskeleton. Méndez-Ferrer et al. described that cyclical
HSC trafficking is regulated by noradrenaline release via the sympathetic nervous system (SNS), transmitted to niche stromal cells by the beta( $\beta)(3)$-adrenergic receptor, resulting in reduced nuclear Sp1 transcription factor and downregulation of CXCL12. There is a close association between so-called Nestin ${ }^{+}$MSC and adrenergic nerve fibers of the SNS and this is believed to control HSC maintenance, egress and functional capacity. ${ }^{15}$ Of additional interest, Maryanovich et al. have recently described how the aging HSC niche is associated with a distinct loss of functional SNS nerve fibers, postulating that denervation-associated remodeling would lead to 'aged' HSC. ${ }^{16}$

Méndez-Ferrer et al. extended their original observations and demonstrated that SNS fibers intrinsically involved in supporting Schwann cells and nestin ${ }^{+}$MSC are reduced in the marrow of MPN patients compared to healthy individuals. ${ }^{14}$ Schwann cell death is initiated following mutant-HSC IL-1 $\beta$ production, and the resultant denervation ultimately leads to a reduction in so-termed 'beneficial MSC' and facilitates expansion of clonal mutant-HSC within the niche. During disease progression, the supportive microenviroment is further disrupted by mutantHSC-mediated hypercytokinemia and thus the MSC population and the osteoblast lineage cells continue to modulate. In the same publication, an MPN murine model was used to demonstrate that restoration of sympathetic regulation of $\mathrm{Nestin}^{+} \mathrm{MSC}$ induced via $\beta_{3}$-agonist exposure abrogated MPN progression and led to a reduction in disease-associated HSC. ${ }^{17}$ The $\beta_{3}$-adrenergic agonist BRL37344 led to reductions in murine BM mutant-HSC-derived progenitors and decreases in neutrophilia, thrombocytosis and marrow fibrosis, associated with a restoration of Nestin ${ }^{+}$MSC. In addition, BRL37344 exposure led to a significant decrease in 'leukemic' stem cells. This pivotal work rapidly led to conceptualization of adrenergic nervous system modulation as a novel therapeutic approach in MPN.

In solid tumor oncogenesis, in vitro studies investigating neuro-biological regulation of tumor establishment, aggressiveness and metastases have emerged over the last five years. ${ }^{18-20}$ By way of example, in contrast to what has been described above in MPN, Magnon et al. studied mice bearing PC-3 prostate tumor xenografts and human prostate tumor specimens, and demonstrated that prostate cancer growth was down-regulated following chemical or surgical sympathectomy or deletion of stromal $\beta_{3}$-adrenergic receptors; tumor samples demonstrated higher densities of adrenergic (surrounding the tumor) and cholinergic (invading the tumor) nerve fibers associated with poorer prognosis, and that the cholinergic parasympathetic nerve fibers were associated with cancer dissemination. ${ }^{20}$ Further links between neuro-modulation and prostate cancer have been suggested by a Norwegian epidemiological study suggesting $\beta$-blocker use was associated with reduced prostate cancer mortality. ${ }^{21}$ Other in vivo work has also investigated the importance of SNS modulation in breast, ovarian and melanoma tumorigenesis, amongst others, and how 


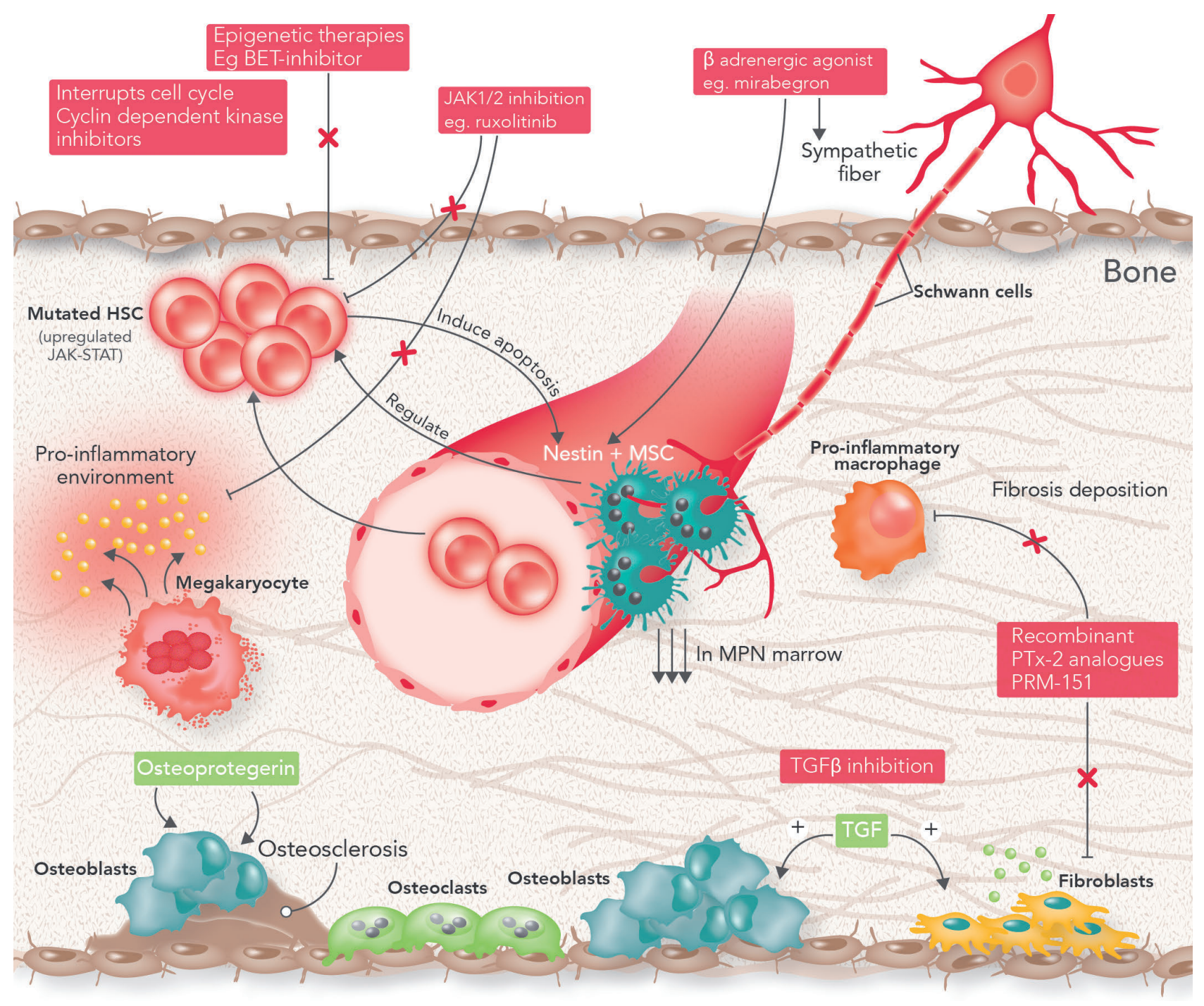

Figure 1. Bone marrow hematopoietic stem cell (HSC) niche in the development and disease maintenance of myeloproliferative neoplasms (MPN). This niche encompasses complex cellular and signaling networks with multiple interactions and 'cross-talk' between HSC, mesenchymal cells (MSC), perivascular cells identified as chemokine ligand 12-abundant reticular cells, osteolineage-derived cells and sinusoidal endothelial cells, amongst others. ${ }^{1.3}$

this is intrinsically linked to both adaptive and innate tumor-immune responses (reviewed by Qiao et al..$^{22}$ ).

In this issue of Haematologica, Drexler et al. report a novel multi-center, phase II trial exploring the refocused use of mirabegron, an oral $\beta-3$ adrenergic agonist with a lower receptor affinity than BRL37344 but commonly used for overactive bladder syndrome. This is the first study in MPN to explore the neural-HSC niche..$^{23}$ Mirabegron (25 $\mathrm{mg}$ titrated to $50 \mathrm{mg}$ once daily) was administered to 39 patients with a diagnosis of MPN fulfilling the World Health Organization (WHO) 2008 diagnostic criteria, and in whom the JAK2-V617F mutant allele burden in granulocyte DNA exceeded $20 \%$ at study entry. The primary end point was defined as the reduction in the JAK2-V617F mutant allele burden of $50 \%$ or more after 24 weeks. A sub-project, in which 20 patients participated, assessed whether mirabegron can restore the Nestin ${ }^{+}$MSC population and alter reticulin fibrosis. The primary end point was not reached in any of the patients, although a $25 \%$ reduction in JAK2-V617F allele burden at 24 weeks was recorded in one patient. Twenty-four percent of patients with polycythemia vera and $29 \%$ of patients with essential thrombocythemia showed hematologic response in accordance with European LeukemiaNet (ELN) and International Working Group Myeloproliferative Neoplasms Research and Treatment (IWG-MRT) criteria. One patient with myelofibrosis became transfusion independent. These clinical findings were considered of minor, possibly insignificant, benefit. Evaluation of BM biopsies showed an increase in Nestin ${ }^{+}$MSC from a median of 1.09 [interquartile range (IOR) $0.38-2.37 / \mathrm{mm}^{2}$ ] to 3.95 (IOR 1.98$\left.8.79 / \mathrm{mm}^{2}\right)(P<0.0001)$, and a slight but significant decrease in reticulin fibrosis from a median grade of 1.0 (IOR 0-3) to 0.5 (IOR 0-2) $(P=0.01)$ between the start and end of mirabegron treatment. The decrease in reticulin fibrosis was only observed in patients not previously treated with 
hydroxycarbamide. A trend was also observed towards reduction in megakaryocyte cluster formation and decrease in numbers of large megakaryocytes with staghorn-like morphology, both cardinal features of MPN. Possible confounding factors are that the participants had established MPN with a median time between MPN diagnosis and trial registration of 3.6 years (range 1.6-8.6 years) and received a relatively short duration of therapy. It is possible that longer exposure to mirabegron, which was well tolerated, and considering its use earlier in the disease course may have demonstrated different results since potentially the chronicity and degree of increase in nestin ${ }^{+}$ MSC may be paramount in ultimately modulating the clinical phenotype. Mirabegron itself, as noted above, is a less potent agent and may have additional effects when compared to BRL37344, which was used in the murine work. Moreover, although significant increases in Nestin ${ }^{+}$MSC were seen, this was limited to those patients not receiving hydroxycarbamide, an agent that most likely affected MSC senescence.

As we begin to explore the complex BM HSC-neurostromal interaction through niche targeting therapies, such as that described in the novel study described above, significant thought needs to be given to the timing of such therapies within the disease course and that logical sequencing / dual agent approaches are considered when targeting of this axis is contemplated. For example, the JAK inhibitor ruxolitinib has been shown to abrogate MSCgrowth and an ability to secrete MCP and IL- 6 which could potentially be beneficial through downregulation of proinflammatory MSC, yet it is unknown what effect prior or concurrent JAK inhibitor therapy would have on $\beta 3$ adrenergic agonist mediated-increases in Nestin ${ }^{+}$MSC. ${ }^{24}$ It is increasingly evident, however, that strategies with the ability to break through the self-perpetuating inflammatory and pro-oncogenic MPN microenvironment are required. Potential combination approaches with drugs acting on this axis to explore include the human pentraxin-2 protein analog PRM-151 (Promedia Pharmaceuticals), which may potentially synergistically reduce fibrosis, or with interferon, which may 'switch on' mutant-HSC-directed immune responses. Both of which are being assessed as single agents and in combination with ruxolitinib (reviewed by Harrison and McLornan ${ }^{25}$ ). Furthermore, an additional benefit of focusing on the stem cell niche could generate a surrogate marker of disease response that would facilitate more rapid evaluation of niche-targeting therapies in the clinical arena. Surrogate markers of disease response moving beyond the blood count, symptoms and spleen are urgently required in this field. An enhanced understanding of the role of the neural network within the MPN niche and how this can be successfully modulated will accelerate the design of such synergistic therapeutic approaches and help the field to move forward. This work from MéndezFerrer and Skoda is also an inspiration in following the academic trail from bench to bedside..$^{13,14,17,23}$

\section{References}

1. Schmitt-Graeff AH, Nitschke R, Zeiser R. The Hematopoietic Niche in Myeloproliferative Neoplasms. Mediators Inflamm. 2015;2015:347270.

2. Schepers K, Campbell T, Passegué E. Normal and leukemic stem cell niches: insights and therapeutic opportunities. Cell Stem Cell.
2015;16(3):254-267

3. Gao X, Xu C, Asada N, Frenette PS. The hematopoietic stem cell niche: from embryo to adult. Development. 2018;145(2).

4. Mead AJ, Mullally A. Myeloproliferative neoplasm stem cells. Blood. 2017;129(12):1607-1616.

5. Zhan H, Lin CHS, Segal Y, Kaushansky K. The JAK2V617F-bearing vascular niche promotes clonal expansion in myeloproliferative neoplasms. Leukaemia. 2018;32(2):462-469.

6. Schepers K, Pietras EM, Reynaud D, et al. Myeloproliferative neoplasia remodels the endosteal bone marrow niche into a self-reinforcing leukemic niche. Cell Stem Cell. 2013;13(3):285-299.

7. Kim YW, Koo BK, Jeong HW, et al. Defective Notch activation in microenvironment leads to myeloproliferative disease. Blood. 2008:112(12):4628-4638.

8. Walkley CR, Olsen GH, Dworkin S, et al. A microenvironmentinduced myeloproliferative syndrome caused by retinoic acid receptor gamma deficiency. Cell. 2007;129(6):1097-1110

9. Wang L, Zhang H, Rodriguez S, et al. Notch-dependent repression of miR-155 in the bone marrow niche regulates hematopoiesis in an NFkB-dependent manner. Cell Stem Cell. 2014;15(1):51-65.

10. Avanzini MA, Bernardo ME, Novara F, et al. Functional and genetic aberrations of in vitro-cultured marrow-derived mesenchymal stromal cells of patients with classical Philadelphia-negative myeloproliferative neoplasms. Leukemia. 2014;28(8):1742-1745.

11. Martinaud C, Desterke C, Konopacki J, et al. Osteogenic Potential of Mesenchymal Stromal Cells Contributes to Primary Myelofibrosis. Cancer Res. 2015;75(22):4753-4765.

12. Ramos TL, Sánchez-Abarca LI, Rosón-Burgo B, et al. Mesenchymal stromal cells (MSC) from JAK2+ myeloproliferative neoplasms differ from normal MSC and contribute to the maintenance of neoplastic hematopoiesis. PLoS One. 2017;12(8):e0182470.

13. Méndez-Ferrer S, Lucas D, Battista M, Frenette PS. Haematopoietic stem cell release is regulated by circadian oscillations. Nature. 2008;452(7186):442-447.

14. Méndez-Ferrer S, Michurina TV, Ferraro F, et al. Mesenchymal and haematopoietic stem cells form a unique bone marrow niche. Nature. 2010; 466(7308):829-834.

15. Katayama Y, Battista M, Kao WM, et al. Signals from the sympathetic nervous system regulate hematopoietic stem cell egress from bone marrow. Cell. 2006;124(2):407-421.

16. Maryanovich $\mathrm{M}$, Zahalka AH, Pierce $\mathrm{H}$, et al. Adrenergic nerve degeneration in bone marrow drives aging of the hematopoietic stem cell niche. Nat Med. 2018;24(6):782-791.

17. Arranz L, Sánchez-Aguilera A, Martín-Pérez D, et al. Neuropathy of haematopoietic stem cell niche is essential for myeloproliferative neoplasms. Nature. 2014;512(7512):78-81.

18. Zahalka AH, Arnal-Estapé A, Maryanovich M, et al. Adrenergic nerves activate an angio-metabolic switch in prostate cancer. Science. 2017;358(6361):321-326.

19. Wolter JK, Wolter NE, Blanch A, et al. Anti-tumor activity of the betaadrenergic receptor antagonist propranolol in neuroblastoma. Oncotarget. 2014;5(1):161-172.

20. Magnon C, Hall SJ, Lin J, et al. Autonomic nerve development contributes to prostate cancer progression. Science. 2013; 341(6142):1236361.

21. Grytli HH, Fagerland MW, Fosså SD, et al. Association between use of $\beta$-bockers and prostate cancer-specific survival: A cohort study of 3561 prostate cancer patients with high-risk or metastatic disease. Eur Urol 2014; 65(3):635-641

22. Oiao G, Chen M, Bucsek MJ, et al. Adrenergic Signaling: A Targetable Checkpoint Limiting Development of the Antitumor Immune Response. Front Immunol. 2018;9:164.

23. Drexler B, Passweg JR, Tzankov A, et al. The sympathomimetic agonist mirabegron did not lower JAK2-V617F allele burden, but restored nestin-positive cells and reduced reticulin fibrosis in patients with myeloproliferative neoplasms: results of phase 2 study SAKK 33/14. Haematologica. 2019;104(4):710-716.

24. Zacharaki D, Ghazanfari R, Li H, et al. Effects of JAK1/2 inhibition on bone marrow stromal cells of myeloproliferative neoplasm (MPN) patients and healthy individuals. Eur J Haematol. 2018;101(1):57-67.

25. Harrison CN, McLornan DP. Current treatment algorithm for the management of patients with myelofibrosis, JAK inhibitors, and beyond. Hematology Am Soc Hematol Educ Program. 2017;2017(1):489-497. 